\title{
Diagnostic accuracy of CBCT versus intraoral imaging for assessment of peri-implant bone defects
}

\author{
Dandan Song $^{1 *} \mathbb{0}$, Sohaib Shujaat ${ }^{1}$, Karla de Faria Vasconcelos ${ }^{1}$, Yan Huang ${ }^{1,2}$, Constantinus Politis ${ }^{1}$, \\ Ivo Lambrichts ${ }^{3}$ and Reinhilde Jacobs ${ }^{1,4}$
}

\begin{abstract}
Background: Early detection of marginal bone loss is vital for treatment planning and prognosis of teeth and implant. This study was conducted to assess diagnostic accuracy of CBCT compared to intra-oral (IO) radiography for detection, classification, and measurement of peri-implant bone defects in an animal model.

Methods: Fifty-four mandible blocks with implants were harvested from nine male health adult beagle dogs with acquisition of IO, CBCT and micro-CT images from all samples. Peri-implant bone defects from 16 samples were diagnosed using micro-CT and classified into 3 defect categories: dehiscence $(n=5)$, infrabony defect $(n=3)$ and craterlike defect $(n=8)$. Following training and calibration of the observers, they asked to detect location (mesial, distal, buccal, lingual) and shape of the defect (dehiscence, horizontal defect, vertical defect, carter-like defect) utilizing both $\mathrm{IO}$ and CBCT images. Both observers assessed defect depth and width on IO, CBCT and micro-CT images at each side of peri-implant bone defect via CT-analyzer software. Data were analyzed using SPSS software and a $p$ value of $<0.05$ was considered as statistically significant.
\end{abstract}

Results: Overall, there was a high diagnostic accuracy for detection of bone defects with CBCT images (sensitivity: 100\%/100\%), while IO images showed a reduction in accuracy (sensitivity: 69\%/63\%). Similarly, diagnostic accuracy for defect classification was significantly higher for CBCT, whereas IO images were unable to correctly identify vestibular dehiscence, with incorrect assessment of half of the infrabony defects. For accuracy of measuring defect depth and width, a higher correlation was observed between CBCT and gold standard micro-CT ( $r=0.91,95 \% \mathrm{Cl} 0.86-0.94)$, whereas a lower correlation was seen for $1 \mathrm{O}$ images $(r=0.82,95 \% \mathrm{Cl} 0.67-0.91)$.

Conclusions: The diagnostic accuracy and reliability of CBCT was found to be superior to IO imaging for the detection, classification, and measurement of peri-implant bone defects. The application of CBCT adds substantial information related to the peri-implant bone defect diagnosis and decision-making which cannot be achieved with conventional IO imaging.

Keywords: Alveolar bone loss, Peri-implantitis, CBCT, Dental radiography

*Correspondence: dandansong9015@gmail.com

${ }^{1}$ OMFS IMPATH Research Group, Department of Imaging and Pathology, Faculty of Medicine, KU Leuven and Oral and Maxillofacial Surgery, University Hospitals Leuven, Kapucijnenvoer 33, 3000 Leuven, Belgium Full list of author information is available at the end of the article

\section{Background}

Peri-implantitis is a pathological inflammatory reaction leading to progressive loss of the supporting bone which exceeds the physiological bone remodeling around an implant [1, 2]. To date, numerous studies have demonstrated the importance of radiographic imaging modalities for the diagnosis of bone 
defects [3-5]. An accurate radiographic assessment of the morphology and size of the peri-implantitis bone defect is of vital clinical importance as it directly influences the implant survival and therapeutic outcome of both surgical and non-surgical defect treatment [6-8].

Two-dimensional (2D) imaging modalities such as intraoral (IO) and panoramic radiography are the most commonly utilized radiographic methods for defect detection in a clinical practice based on their low radiation exposure and cost-effectiveness [9-13]. However, they have certain limitations such as, 2D representation of three-dimensional (3D) anatomical structures, geometric distortion, lower spatial resolution, and image magnification which underestimates the defect [14-17]. Furthermore, their inability to diagnose and distinguish buccally and lingually located defects may lead to an inaccurate representation of the bone defect $[18,19]$. To overcome the limitations associated with 2D radiography, cone beam computed tomography (CBCT) has been proposed and recommended by various studies as a modality of choice for assessment of periodontal bone defects [20-23]. Undoubtedly, CBCT offers higher accuracy compared to its 2D counterparts for an earlier bone defect detection, thereby allowing immediate application of interventions for controlling further bone loss. Nevertheless, only a few studies are available assessing the superiority of CBCT over $2 \mathrm{D}$ imaging for the assessment of peri-implant bone defects $[23,24]$.

Furthermore, microscopic computed tomography (micro-CT), one of the most versatile non-invasive investigative techniques has been regarded as a standard tool for quantifying the density and architecture of bone in preclinical investigations. Micro-CT functions by illuminating a rotated object with $\mathrm{x}$-rays and collects the magnified projection images via planar $\mathrm{x}$-ray detector. Thereafter, multiple angular images are obtained and stacked together to form the 3D image. Henceforth, micro-CT not only has the ability of imaging the internal biological structures without the need for sample preparation but also provides with $3 \mathrm{D}$ representation of the anatomical structures. Previous studies have widely reported on the feasibility and reliability of using micro-CT to evaluate morphologic characteristics of cortical and trabecular bone in both animal and human models [25-28].

Therefore, the current study was conducted to assess the diagnostic accuracy of CBCT compared to IO radiography using the micro-CT as the standard for the detection, classification, and measurement of periimplant bone defects in an animal model.

\section{Methods}

Following ethical approval from the Bioethics Committee of Sichuan University (Reference No: WCCSIRBD-2014-010), nine health male adult beagle dogs (weight 14-17 kg, age 12-14 months) with completely sound oral condition were recruited by following the ARRIVE guidelines [29] for preclinical animal studies (Supplementary file). All animals were provided by Laboratory Animal Center of Sichuan University. An identical housing and feeding condition was required for all the animals at the Experimental Animal Center of Laboratory of Biotherapy. With injecting general anesthesia with Sumianxin $(0.1 \mathrm{ml} / \mathrm{kg}$ xylazine hydrochloride, Changchun Military Academy of Medical Sciences, Changchun, China) and local anesthesia (2-4 ml lidocaine $2 \%$ epinephrine, Tianjin Pharmaceutical Co. Ltd, Tianjin, China) at the surgical sites, a total of 54 screw-type titanium dental implant with plasma-sprayed hydroxyapatite (HA) coating (3.3 $\mathrm{mm} \varnothing \times 8 \mathrm{~mm}$, cylindrical, non-submerged healing, BLB, China) were inserted in the mandibular region of each $\operatorname{dog}(n=6$ per $\operatorname{dog})$. The sample size was calculated based on a prior power analysis in $G^{*}$ power 3.1 at a power of $80 \%$ [30]. Following crown preparation and attachment, each implant was followed-up for a period of at least 1 months. The surgical procedure has been explained in a prior publication [31, 32]. Thereafter, all animals were euthanized using an overdose of xylazine hydrochloride (intravenous injection) and immediate perfusion of $4 \%$ paraformaldehyde and $0.0125 \%$ glutaraldehyde in $0.1 \mathrm{M}$ phosphate buffer ( $\mathrm{pH}$ 7.4). All dogs were healthy with clinically stable implants before sacrifice. The mandible blocks with implants were harvested and $\mathrm{IO}, \mathrm{CBCT}$ and micro-CT images were acquired for each sample, where micro-CT acted as the gold standard. Table 1 describes the details of the acquisition devices and scanning parameters. Following image acquisition, 16 samples were found to have bone defects and were included in the study. The marginal bone level around the implant lower than the first screw loop of the implant from the top was judged as the bone defect; otherwise, the others were excluded. All the images were manually reoriented along implants long axis with DataViewer (ver. 1.5.1.2, Bruker). Following orientation, three types of bone defects were recognized and diagnosed using micro-CT, which included dehiscence $(n=5)$, infrabony defect $(n=3)$ and crater-like defect $(n=8)$ (Fig. 1). The diagnosis was carried out by a consultant oral and maxillofacial radiologist with an experience of over 20 years. Later, two dentists were recruited as observers with an experience of at least 5 years in dental imaging. Following training and calibration of the observers, all the samples marked by the implant site were renumbered and randomized by the method of random sort in Excel. The 
Table 1 Details of the protocols for each acquisition device and scanning parameter

\begin{tabular}{lllr}
\hline & Intraoral radiography & Cone-beam computed tomography & Micro-CT \\
\hline Product name & Heliodent Plus & 3D Accuitomo 170 & Quantum FX \\
Company & Sirona Dental Systems GmbH & J. Morita & PerkinElmer, Inc. \\
& Bensheim, Germany & Waltham, USA \\
Tube current $(\mathrm{mA})$ & 7 & 5 & 0.16 \\
Tube voltage $(\mathrm{kV})$ & 60 & 90 & 90 \\
Voxel size $(\mathrm{mm})$ & - & 0.08 & 0.02 \\
Field of view $(\mathrm{cm})$ & $3.3 \times 4.3$ & $10 \times 5$ & $0.01 \times 0.01$ \\
Exposure time $(\mathrm{s})$ & 0.12 & 17.5 & 180 \\
\hline
\end{tabular}

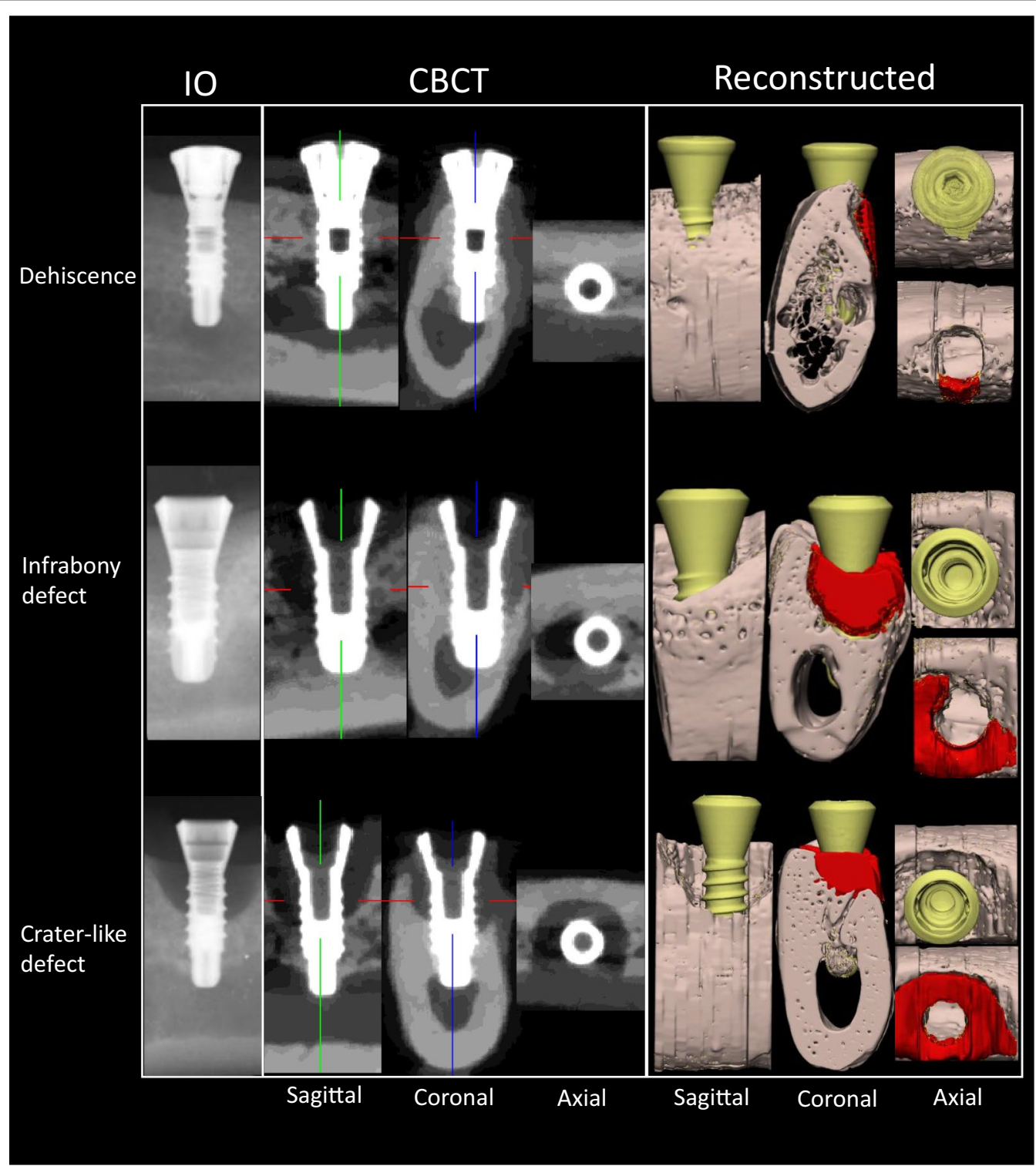

Fig. 1 Shapes of peri-implant bone defects which were demonstrated in IO, CBCT and reconstructed imaging 
observers were asked to detect the location of the defect (mesial, distal, buccal, lingual) and the shape of the defect (dehiscence, horizontal defect, vertical defect, crater-like defect). All evaluations were performed with both IO and CBCT images.

Following diagnosis, both observers measured the defect depth and width on IO, CBCT and micro-CT images at each side of peri-implant bone defect via CTanalyzer software (version 1.16.1.0, Skyscan1272, Bruker MicroCT, Kontich, Belgium). The slices in 3D images were standardized to that of the $2 \mathrm{D}$ image. A mesio-distal and bucco-lingual slice was selected from the sagittal and coronal view individually on the 3D images and were oriented parallel to the long axis of implant. The width and depth of the defects were measured as shown in Fig. 2. The observers performed both diagnosis and measurement tasks at a two weak interval with randomization of the data for assessing the observer reliability.

\section{Statistical analysis}

Data were analyzed using SPSS software (Version 22, IBM, New York, USA). The diagnostic accuracy of IO and $\mathrm{CBCT}$ images for the defect detection and classification was assessed by calculating the sensitivity and intraand inter-rater reliability (Cohen's and Fleiss's Kappa) of each method and observer. The interpretation of Kappa values was carried out as suggested by Landis and Koch [33]. Intra-class correlation coefficient (ICC) was utilized for calculating the absolute inter-protocol, intra-rater, and inter-rater agreement for defects depth and width measurements. Kruskal-Wallis one-way ANOVA test was performed to compare the depth and width of the infrabony defect with that of the crater-like defect. A $p$ value of $<0.05$ was considered as statistically significant.

\section{Results}

Overall, a high agreement (Kappa: 0.92; 95\% CI 0.880.96 ) and reliability (ICC: $0.97 ; 95 \%$ CI $0.96-0.97$ ) was observed for the detection and classification of the defect on IO and CBCT images. Intra- and inter-rater agreement for the diagnosis with $\mathrm{CBCT}$ was found to be almost perfect for each observer. The reliability of CBCT images was also considerably high when compared with the micro-CT for the detection and classification of the bone defect (ICC: 0.93 and 0.96, 95\% CI 0.9-0.95 \& 0.95-0.98). Additionally, the intra-rater and inter-rater agreement between both observers was higher with CBCT compared to IO images (Table 2). Both observers showed a high diagnostic accuracy for detection of the bone defect with CBCT images (sensitivity: 100\%/100\%), whereas, IO images showed a reduction in the accuracy (sensitivity: 69\%/63\%). Similarly, the diagnostic accuracy for the classification of the defect was also higher for CBCT when compared with IO images (Table 3).

For the accuracy of measuring depth and width of the defect, the ICC and agreement with the gold-standard micro-CT was found to be approximately 1 (ICC: 0.99 , 95\% CI 0.996-0.998; Kappa: 0.91). A higher correlation

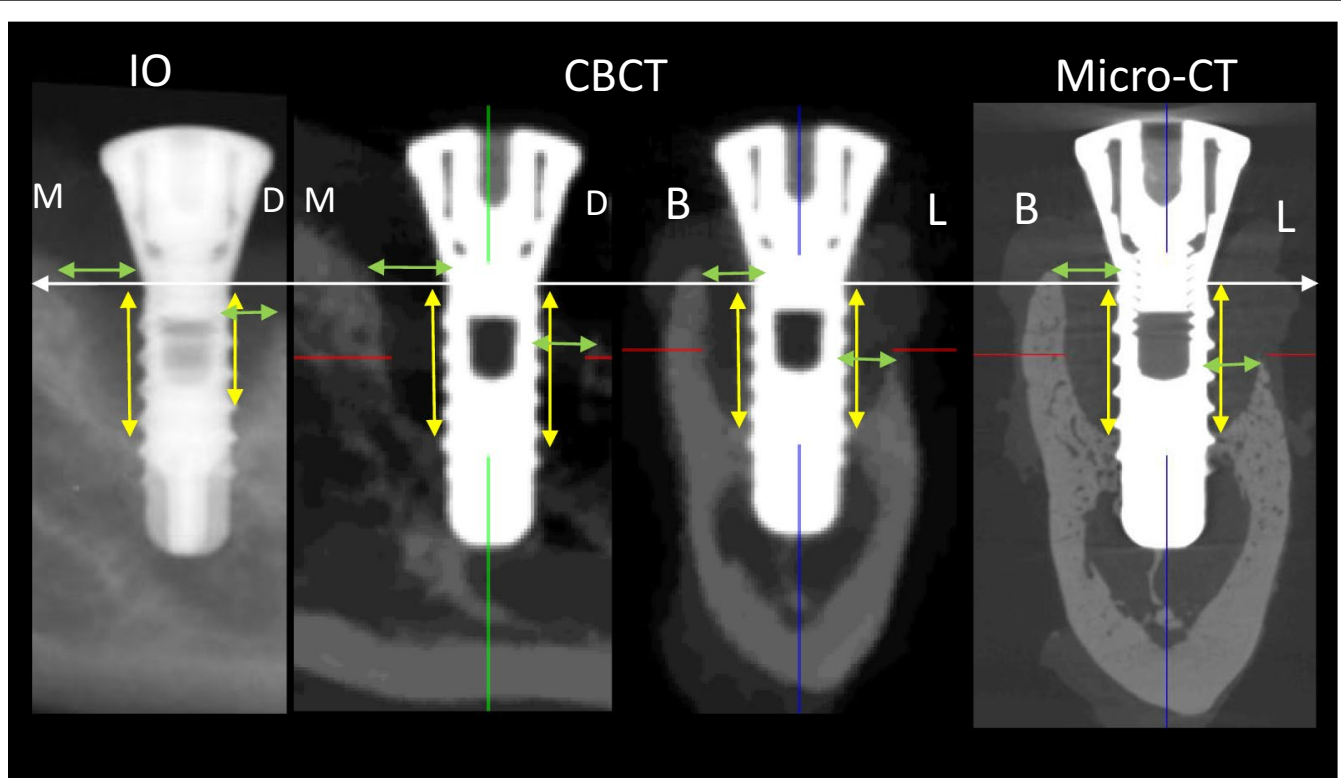

Fig. 2 Method of depth and width measurement in intra-oral radiography (IO), cone-beam CT (CBCT) and micro-CT imaging. White arrow, implant shoulder as reference; Yellow arrow, depth of bone defect (from implant shoulder to the most apical of bone defect); Green arrow, width of bone defect (from implant shoulder to bone crest) 
Table 2 Reliability and agreement in detection of the bone lesion and morphology classification

\begin{tabular}{|c|c|c|c|c|c|}
\hline \multirow[t]{3}{*}{ Detection parameters } & \multirow[t]{3}{*}{ Methods } & \multicolumn{4}{|c|}{ Observer effect } \\
\hline & & \multicolumn{2}{|c|}{ Reliability } & \multirow{2}{*}{$\begin{array}{l}\text { Agreement } \\
\text { Weighted Kappa }\end{array}$} & \multirow[b]{2}{*}{$95 \% \mathrm{Cl}$} \\
\hline & & ICC & $95 \% \mathrm{Cl}$ & & \\
\hline \multirow[t]{3}{*}{ Bone defect presence } & 10 & 0.96 & $0.94-0.97$ & 0.84 & $0.72-0.96$ \\
\hline & $\mathrm{CBCT}$ & 0.97 & $0.96-0.98$ & 0.87 & $0.74-0.99$ \\
\hline & Total & 0.90 & $0.86-0.92$ & 0.78 & $0.68-0.88$ \\
\hline \multirow[t]{3}{*}{ Shape classification } & 10 & 0.96 & $0.94-0.97$ & 0.84 & $0.72-0.94$ \\
\hline & $\mathrm{CBCT}$ & 1 & - & 0.94 & $0.97-1$ \\
\hline & Total & 0.92 & $0.90-0.94$ & 0.82 & $0.74-0.90$ \\
\hline Total & & 0.97 & $0.96-0.97$ & 0.92 & $0.88-0.96$ \\
\hline
\end{tabular}

Reliability, intra-class correlation coefficient; Agreement, inter-rater agreement; IO, intra-oral radiography; CBCT, cone beam computer tomography

Table 3 Sensitivity of the diagnosis of bone defect presence and shape

\begin{tabular}{|c|c|c|c|c|c|}
\hline \multirow[t]{2}{*}{ Observers } & \multirow[t]{2}{*}{ Methods } & \multirow{2}{*}{$\begin{array}{l}\text { Bone } \\
\text { defect } \\
\text { presence } \\
(\%)\end{array}$} & \multicolumn{3}{|c|}{ Bone defect shape } \\
\hline & & & Dehiscence & $\begin{array}{l}\text { Infrabony } \\
\text { defect } \\
(\%)\end{array}$ & $\begin{array}{l}\text { Crater- } \\
\text { like } \\
\text { defect } \\
(\%)\end{array}$ \\
\hline \multirow[t]{2}{*}{1} & 10 & 69 & 0 & 50 & 78 \\
\hline & $\mathrm{CBCT}$ & 100 & $100 \%$ & 100 & 90 \\
\hline \multirow{2}{*}{2} & 10 & 63 & 0 & 50 & 78 \\
\hline & $\mathrm{CBCT}$ & 100 & $100 \%$ & 100 & 89 \\
\hline
\end{tabular}

$\mathrm{IO}$, intra-oral radiography, $\mathrm{CBCT}$, cone beam computer tomography

was observed when CBCT was compared with microCT $(r=0.91,95 \%$ CI 0.86-0.94), whereas slightly lower correlation was seen with IO images $(r=0.82,95 \%$ CI $0.67-0.91)$.

The relationship between the bone defect morphology and size is demonstrated in Fig. 3. The depth for craterlike defect was larger than infrabony defect at all sides in both IO and CBCT images. The distal $(p=0.003)$ and buccal side $(p=0.002)$ showed a significantly larger depth in crater-like defects. Similarly, a larger defect width was observed for crater-like defects in both IO and CBCT images, except mesial side on CBCT images which showed a larger width in infrabony defect. Whereas, IO images showed a significantly larger width on the mesial side of the crater-like defect.

\section{Discussion}

Evidence suggests multiple studies underlining the importance of the accurate detection and classification of bone defect which can influence the treatment planning and prognosis of dental implant [34-36]. Therefore, the present study was conducted to assess the diagnostic accuracy of CBCT and IO imaging for the detection, classification, and measurement of peri-implant bone defects.

Our findings suggested a higher reliability and diagnostic accuracy for the detection and classification of peri-implant defects with CBCT compared to IO images. Based on the 2D nature of IO radiographs, it only allowed accurate diagnosis of the horizontal and 1-wall vertical bone defects in a mesiodistal direction [5]. This could have led to a higher sensitivity of $\mathrm{CBCT}$ which offers $3 \mathrm{D}$ visualization of the bone. These findings were consistent with previous studies, which suggested $\mathrm{CBCT}$ to have a significantly higher diagnostic accuracy compared to digital periapical radiography for the detection of fenestration, dehiscence and three-walled periodontal defects $[23,24,37]$. In a study by Noujeim, et al. [22], a high sensitivity was recorded for the detection of large bone defects by both IO and CBCT imaging and they suggested $\mathrm{CBCT}$ imaging only for small bone defects of $<1 \mathrm{~mm}$ where IO radiography was considered insufficient for an accurate diagnosis. However, their study has limited clinical value as they observed defects only in the mesiodistal direction, and buccolingual aspect of the defect was not included in the evaluation. Whereas, in our study five bone defects were with dehiscence on the lingual side which could not be detected or classified accurately on IO radiographs, thereby leading to a further reduction in its diagnostic accuracy [5]. Similarly, inconsistency was observed with a study by Kühl, et al. [16]. where authors found that IO radiography offered improved accuracy compared to CT-based images for the detection of peri-implant bone defect. The main reason for decreased accuracy of CT-based images in their study was based on the presence metal artefacts resulting in lower quality images. On the other hand, we acquired images with a high-resolution $\mathrm{CBCT}$ protocol and the CBCT device had an inbuilt metal artefact reduction algorithm resulting in high quality images. 
10

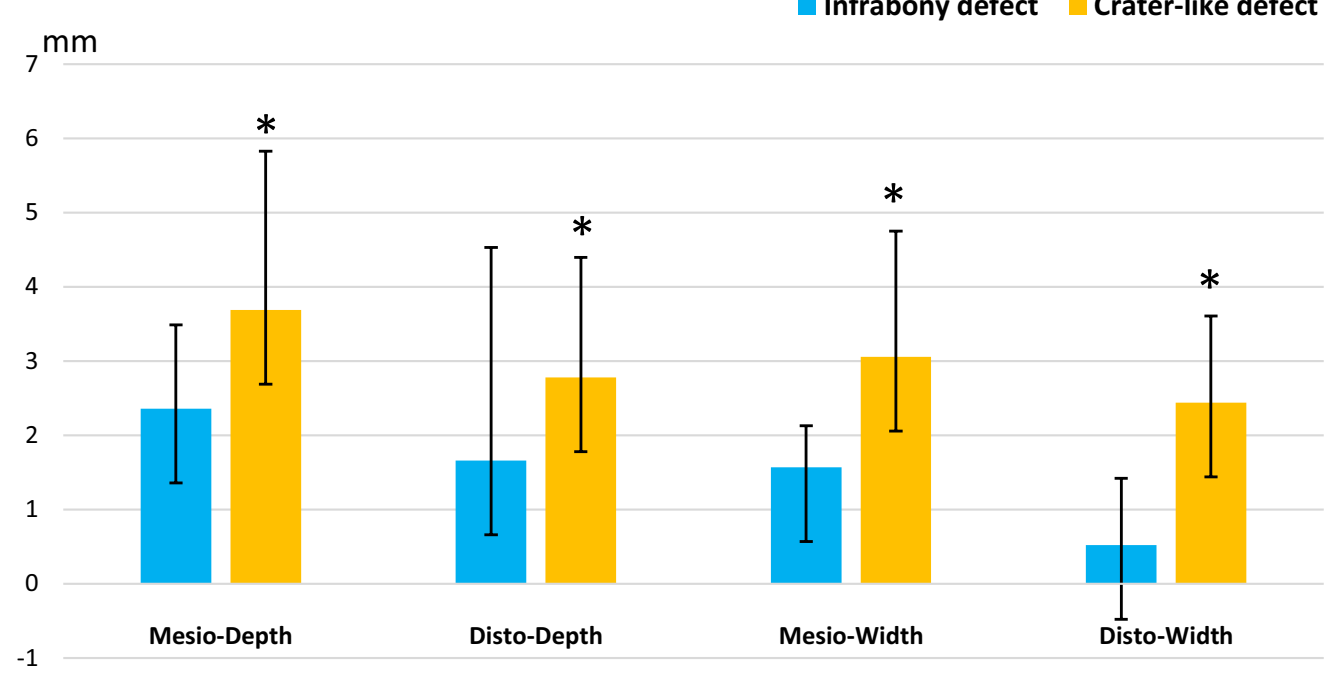

$-1$

CBCT

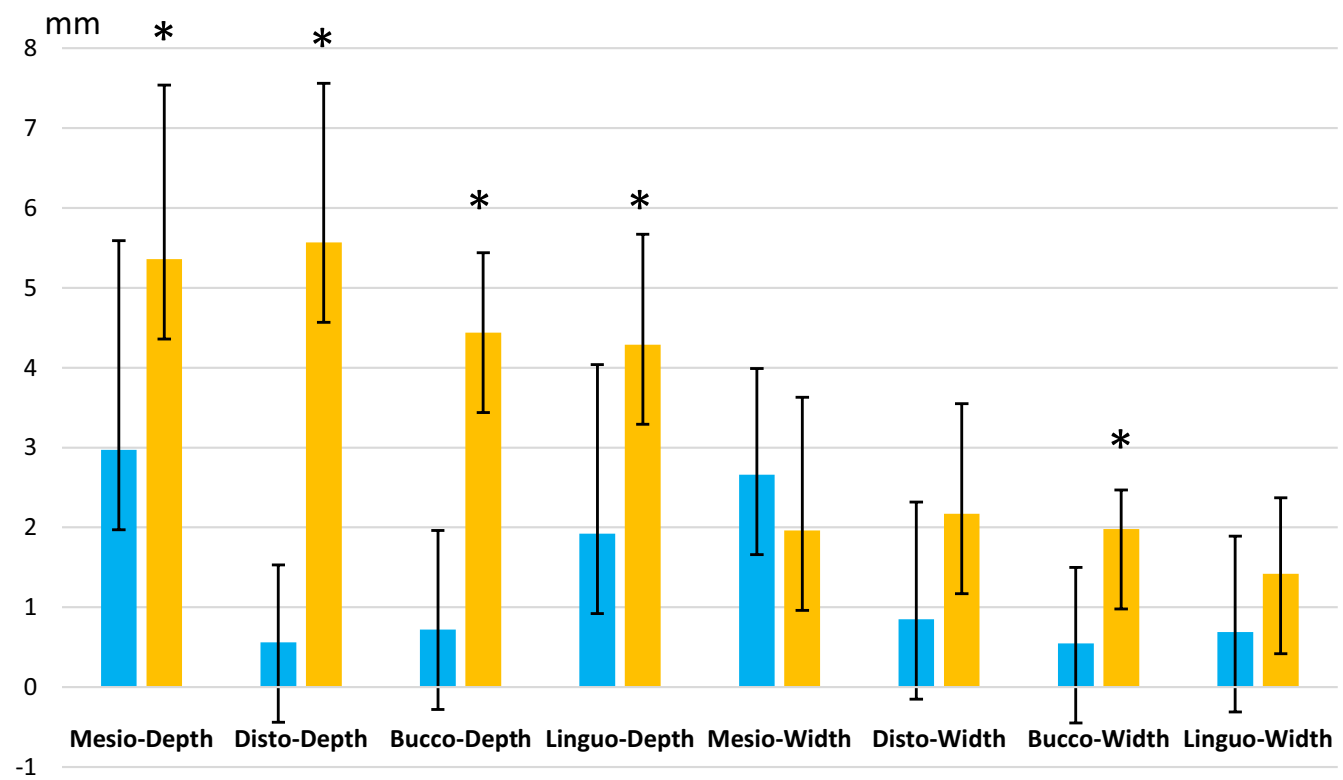

Fig. 3 Graph of bone defect size (depth and width) for different shapes of peri-implant bone lesion in IO and CBCT imaging. ${ }^{*} p<0.05$. IO: Intra-oral radiography; CBCT: cone beam computer tomography

In relation to the mesiodistal depth and width measurements of the bone defect, a high correlation was found for both IO $(r=0.82)$ and CBCT $(r=0.92)$ images when compared with the micro-CT. The slightly lower correlation value with IO could have resulted due to the overlapping effect and blurring of the peri-implant region. We found the size of defect to be significantly larger in crater-like defect compared to infrabony defect. A previous study observed peri-implant bone defects to be larger on buccal side compared to other walls in patients with peri-implantitis [38]. In contrast, we found defects to be larger mesiodistally followed by lingual and buccal wall. The difference in study design where we utilized an animal model and location of the implant placement could have resulted in these inconsistent findings. The detection, classification and accurate measurements of the defect are all critical parameters as they might influence the success of an implant or regenerative therapy if required [39]. Although $\mathrm{CBCT}$ carries the risk of higher radiation dose compared to IO radiography, it can be 
argued whether the benefit of an accurate diagnosis with CBCT outweighs the risks involved with a higher radiation dose. We believe that a clinician should not hesitate to order a CBCT for the diagnosis and follow-up of a peri-implant defect, as IO radiography is insufficient for an accurate diagnosis [40]. At the same instance, the scanning parameters should be optimized accordingly for reducing the radiation exposure to the patient.

Our study had certain limitations. Firstly, the sample size was not sufficient for drawing a meaningful conclusion related to the relationship between the bone defect type and size. Secondly, the setup for the high resolution CBCT was obviously diverting from that of a normal patient acquisition. We realise that this may cause some deviation when transferring the present imaging data and outcome to the clinic situation. However, the highdose allowed accurate investigation of infra-bony defects which might get impeded due to the presence of implantrelated artefacts if a lower dose is applied. Further studies are required to assess the diagnostic accuracy of lowdose CBCT protocols in patients for detecting and classifying peri-implant bone defects. In the midst of these limitations, we provided evidence related to the detection of bone defects which might not be visible on IO radiographs, thereby leading to an inaccurate diagnosis and treatment planning. Although IO radiography is considered as a clinical standard for assessing peri-implant bone defects [41], the importance of CBCT imaging for diagnosis and follow-up should not be ignored for employing timely management of the defect $[42,43]$. Further studies should be carried out with patient specific low-dose CBCT protocols to assess their accuracy for peri-implant bone defect diagnosis. Also, future studies should pay attention to the implant blooming artefacts that are impacting peri-implant visibility and are influenced by CBCT device and protocol characteristics as well as by implant material and design $[44,45]$.

\section{Conclusions}

The diagnostic accuracy and reliability of $\mathrm{CBCT}$ was found to be superior to IO imaging for the detection, classification, and measurement of peri-implant bone defects. The application of CBCT adds substantial information related to the peri-implant bone defect diagnosis and decision-making which cannot be achieved with conventional IO imaging. However, benefit-risk ratio should be kept in mind and CBCT should be acquired for cases where a peri-implant bone defect might influence the implant survival rate.

\section{Abbreviations}

CBCT: Cone beam computed tomography; Micro-CT: Microscopic computed tomography; IO: Intraoral radiography; 2D: Two-dimensional; 3D:
Three-dimensional; HA: Plasma-sprayed hydroxyapatite; ICC: Intra-class correlation coefficient.

\section{Acknowledgements}

The authors are thankful to the laboratory technicians from the Research Base of West China Hospital, Sichuan University and Translational Cell and Tissue Research of University Hospitals Leuven, KU Leuven for their valuable help during this animal study.

\section{Authors' contribution}

$C P, I L$, and RJ designed this study. HY did the animal experiment. DS and KFV performed the measurements. DS and SS wrote the first draft of the manuscript. SS and RJ corrected the manuscript. All authors have read and approved the final manuscript

\section{Funding}

DS received fellowship support from China Scholarship Council. (201708210187). The funding plays no role in the design of the study and collection, analysis, and interpretation of data and in writing the manuscript.

\section{Availability of data and materials}

The datasets used and/or analyzed during the current study are available from the corresponding author on reasonable request.

\section{Ethics approval and consent to participate}

This animal experiment was approved by the Bioethics Committee of Sichuan University (reference number: WCCSIRB-D-2014-010).

\section{Consent for publication}

Not applicable.

\section{Competing interests}

The authors declare that they have no competing interests.

\section{Author details}

1 OMFS IMPATH Research Group, Department of Imaging and Pathology, Faculty of Medicine, KU Leuven and Oral and Maxillofacial Surgery, University Hospitals Leuven, Kapucijnenvoer 33, 3000 Leuven, Belgium. ${ }^{2}$ West China College of Stomatology, State Key Laboratory of Oral Disease \& National Clinical Research Center for Oral Disease, Sichuan University, Chengdu, China. ${ }^{3}$ Department of Morphology, Biomedical Research Institute, Hasselt University, Diepenbeek, Belgium. ${ }^{4}$ Department of Dental Medicine, Karolinska Institute, Stockholm, Sweden.

Received: 13 January 2021 Accepted: 1 February 2021

Published online: 10 February 2021

\section{References}

1 Zitzmann NU, Berglundh T. Definition and prevalence of peri-implant diseases. J Clin Periodontol. 2008;35(Suppl 8):286-91.

2. Berglundh T, Zitzmann NU, Donati M. Are peri-implantitis lesions different from periodontitis lesions? J Clin Periodontol. 2011;38(Suppl 11):188-202.

3. Schwarz F, Herten M, Sager M, Bieling K, Sculean A, Becker J. Comparison of naturally occurring and ligature-induced peri-implantitis bone defects in humans and dogs. Clin Oral Implants Res. 2007:18:161-70.

4. Harris D, Horner K, Gröndahl K, Jacobs R, Helmrot E, Benic Gl, et al. E.A.O. guidelines for the use of diagnostic imaging in implant dentistry 2011. A consensus workshop organized by the European Association for Osseointegration at the Medical University of Warsaw. Clin Oral Implants Res. 2012:23:1243-53.

5 Hilgenfeld T, Juerchott A, Deisenhofer UK, Krisam J, Rammelsberg P, Heiland S, et al. Accuracy of cone-beam computed tomography, dental magnetic resonance imaging, and intraoral radiography for detecting peri-implant bone defects at single zirconia implants---an in vitro study. Clin Oral Implants Res. 2018;29:922-30.

6. Tözüm TF, Turkyilmaz I, Yamalik N, Karabulut E, Türkyilmaz AS, Eratalay K. Analysis of the possibility of the relationship between various implant-related measures: an 18-month follow-up study. J Oral Rehabil. 2008;35:95-104. 
7. Merheb J, Coucke W, Jacobs R, Naert I, Quirynen M. Influence of bony defects on implant stability. Clin Oral Implants Res. 2010;21:919-23.

8. Tsitoura E, Tucker R, Suvan J, Laurell L, Cortellini P, Tonetti M. Baseline radiographic defect angle of the intrabony defect as a prognostic indicator in regenerative periodontal surgery with enamel matrix derivative. J Clin Periodontol. 2004;31:643-7.

9 Papapanou PN, Tonetti MS. Diagnosis and epidemiology of periodontal osseous lesions. Periodontology 2000. 2000;22:8-21.

10. Tugnait A, Clerehugh V, Hirschmann PN. The usefulness of radiographs in diagnosis and management of periodontal diseases: a review. J Dent. 2000;28:219-26.

11. Tugnait A, Clerehugh V, Hirschmann PN. Use of the basic periodontal examination and radiographs in the assessment of periodontal diseases in general dental practice. J Dent. 2004;32:17-25.

12. Esmaeli F, Shirmohammadi A, Faramarzie M, Abolfazli N, Rasouli H, Fallahi S. Determination of vertical interproximal bone loss topography: correlation between indirect digital radiographic measurement and clinical measurement. Iran J Radiol. 2012;9:83-7.

13 De Bruyn H, Vandeweghe S, Ruyffelaert C, Cosyn J, Sennerby L. Radiographic evaluation of modern oral implants with emphasis on crestal bone level and relevance to peri-implant health. Periodontology 2000. 2013:62:256-70.

14. Jeffcoat MK, Wang IC, Reddy MS. Radiographic diagnosis in periodontics. J Periodontol. 1995;7:54-68.

15. Eickholz P, Kim T, Benn D, Staehle H. Accuracy of radiographic assessments of interproximal bone loss. Oral Surg Oral Med Oral Pathol Oral Radiol Oral Endod. 1998:85:99-106.

16 Kühl S, Zürcher S, Zitzmann NU, Filippi A, Payer M, Dagassan-Berndt D. Detection of peri-implant bone defects with different radiographic techniques---a human cadaver study. Clin Oral Implants Res. 2016;27:529-34.

17 Ritter L, Elger MC, Rothamel D, Fienitz T, Zinser M, Schwarz F, et al. Accuracy of peri-implant bone evaluation using cone beam CT, digital intra-oral radiographs and histology. Dentomaxillofac Radiol. 2014;43:20130088.

18. Rees TD, Biggs NL, Collings CK. Radiographic interpretation of periodontal osseous lesions. Oral Surg Oral Med Oral Pathol. 1971;32:141-53.

19. Schwarz F, Sahm N, Schwarz K, Becker J. Impact of defect configuration on the clinical outcome following surgical regenerative therapy of periimplantitis. J Clin Periodontol. 2010;37:449-55.

20. Vandenberghe $B$, Jacobs R, Yang J. Diagnostic validity (or acuity) of $2 D$ CCD versus 3D CBCT-images for assessing periodontal breakdown. Oral Surg Oral Med Oral Pathol Oral Radiol Endod. 2007:104:395-401.

21. Braun $X$, Ritter $L$, Jervøe-Storm PM, Frentzen M. Diagnostic accuracy of CBCT for periodontal lesions. Clin Oral Investig. 2014;18:1229-36.

22 Noujeim M, Prihoda T, Langlais R, Nummikoski P. Evaluation of high-resolution cone beam computed tomography in the detection of simulated interradicular bone lesions. Dentomaxillofac Radiol. 2009;38:156-62.

23 Bayat S, Talaeipour AR, Sarlati F. Detection of simulated periodontal defects using cone-beam CT and digital intraoral radiography. Dentomaxillofac Radiol. 2016:45:20160030.

24. Christiaens V, De Bruyn H, De Vree H, Lamoral S, Jacobs R, Cosyn J. A controlled study on the accuracy and precision of intraoral radiography in assessing interproximal bone defect morphology around teeth and implants. Eur J Oral Implantol. 2018;11:361-7.

25. Bonnet N, Laroche N, Vico L, Dolleans E, Courteix D, Benhamou CL. Assessment of trabecular bone microarchitecture by two different $\mathrm{x}$-ray microcomputed tomographs: a comparative study of the rat distal tibia using Skyscan and Scanco devices. Med Phys. 2009;36:1286-97.

26 Bouxsein ML, Boyd SK, Christiansen BA, Guldberg RE, Jepsen KJ, Müller R. Guidelines for assessment of bone microstructure in rodents using micro-computed tomography. J Bone Miner Res. 2010;25(7):1468-86.

27. Arvidsson A, Sarve H, Johansson CB. Comparing and visualizing titanium implant integration in rat bone using 2D and 3D techniques. J Biomed Mater Res Part B. 2015:103:12-20.

28 Van Dessel J, Nicolielo LF, Huang Y, Slagmolen P, Politis C, Lambrichts I, et al. Quantification of bone quality using different cone beam computed tomography devices: accuracy assessment for edentulous human mandibles. Eur J Oral Implantol. 2016:9:411-24.

29 Percie du Sert N, Hurst V, Ahluwalia A, Alam S, Avey MT, Baker M, et al. The ARRIVE guidelines 2.0: updated guidelines for reporting animal research. BMC Vet Res. 2020;16:242

30 Faul F, Erdfelder E, Lang A-G, Buchner A. G* Power 3: a flexible statistical power analysis program for the social, behavioral, and biomedical sciences. Behav Res Methods. 2007;39:175-91.

31. Song D, Huang Y, Van Dessel J, Shujaat S, Orhan K, Vangansewinkel T, et al. Effect of platelet-rich and platelet-poor plasma on peri-implant innervation in dog mandibles. Int J Implant Dent. 2019;5:40.

32 Huang Y, Li Z, Van Dessel J, Salmon B, Huang B, Lambrichts I, et al. Effect of platelet-rich plasma on peri-implant trabecular bone volume and architecture: a preclinical micro-CT study in beagle dogs. Clin Oral Implants Res. 2019;30:1190-9.

33. Landis JR, Koch GG. An application of hierarchical kappa-type statistics in the assessment of majority agreement among multiple observers. Biometrics. 1977;33:363-74.

34. Oliveira Costa F, Cota LO, Costa JE, Pordeus IA. Periodontal disease progression among young subjects with no preventive dental care: a 52-month follow-up study. J Periodontol. 2007;78:198-203.

35. Cortellini P, Prato GP, Tonetti MS. Periodontal regeneration of human infrabony defects. I. Clinical measures. J Periodontol. 1993;64:254-60.

36. Tonetti MS, Prato GP, Williams RC, Cortellini P. Periodontal regeneration of human infrabony defects. III. Diagnostic strategies to detect bone gain. J Periodontol. 1993;64(4):269-77.

37 Vandenberghe B, Jacobs R, Yang J. Detection of periodontal bone loss using digital intraoral and cone beam computed tomography images: an in vitro assessment of bony and/or infrabony defects. Dentomaxillofac Radiol. 2008;37:252-60.

38 Monje A, Insua A, Rakic M, Nart J, Moyano-Cuevas JL, Wang HL. Estimation of the diagnostic accuracy of clinical parameters for monitoring peri-implantitis progression: an experimental canine study. J Periodontol. 2018;89:1442-51.

39 Bender P, Salvi GE, Buser D, Sculean A, Bornstein MM. Correlation of three-dimensional radiologic data with subsequent treatment approach in patients with peri-implantitis: a retrospective analysis. Int J Periodontics Restor Dent. 2017;37:481-9.

40. Eickholz P, Hausmann E. Accuracy of radiographic assessment of interproximal bone loss in intrabony defects using linear measurements. Eur J Oral Sci. 2000;108:70-3.

41. Pepelassi EA, Tsiklakis K, Diamanti-Kipioti A. Radiographic detection and assessment of the periodontal endosseous defects. J Clin Periodontol. 2000;27:224-30.

42. Guerrero ME, Jacobs R, Loubele M, Schutyser F, Suetens P, van Steenberghe D. State-of-the-art on cone beam CT imaging for preoperative planning of implant placement. Clin Oral Investig. 2006;10:1-7.

43. Maki K, Inou N, Takanishi A, Miller AJ. Computer-assisted simulations in orthodontic diagnosis and the application of a new cone beam X-ray computed tomography. Orthodont Craniofac Res. 2003;6(Suppl 1):95-101. discussion 79-82.

44. Codari M, de Faria Vasconcelos K, Ferreira Pinheiro Nicolielo L, Haiter Neto $\mathrm{F}$, Jacobs R. Quantitative evaluation of metal artifacts using different CBCT devices, high-density materials and field of views. Clin Oral Implants Res. 2017:28:1509-14.

45. Vanderstuyft T, Tarce M, Sanaan B, Jacobs R, de Faria Vasconcelos K, Quirynen M. Inaccuracy of buccal bone thickness estimation on conebeam CT due to implant blooming: An ex-vivo study. J Clin Periodontol. 2019;46:1134-43.

\section{Publisher's note}

Springer Nature remains neutral with regard to jurisdictional claims in published maps and institutional affiliations. 